\title{
A longitudinally extensive H3 K27M-mutant diffuse midline glioma in an elderly patient clinically mimicking central nervous system inflammation: a case report
}

\author{
Kristof Babarczy ${ }^{1}$, Zita Reisz ${ }^{2,3}$, Elza Szabo ${ }^{1}$, Cecilia Rajda ${ }^{1}$, Laszlo Vecsei ${ }^{1,4}$, Istvan Bodi ${ }^{3}$, Peter Klivenyi ${ }^{1}$, \\ Tibor Hortobagyi ${ }^{2}$, Levente Szalardy ${ }^{1}$ \\ ${ }^{1}$ Department of Neurology, Faculty of Medicine, Albert Szent-Györgyi Clinical Center, University of Szeged, Szeged, Hungary, ${ }^{2}$ Department \\ of Pathology, Faculty of Medicine, Albert Szent-Györgyi Clinical Center, University of Szeged, Szeged, Hungary, ${ }^{3}$ Department of Clinical \\ Neuropathology, King's College Hospital, London, United Kingdom, ${ }^{4}$ MTA-SZTE Neuroscience Research Group, Szeged, Hungary
}

\begin{abstract}
Diffuse midline gliomas, H3 K27M-mutant, World Health Organization (WHO) grade IV represent a distinct glioma entity with a predominantly paediatric presentation and remarkably poor prognosis. This report presents a case of a 73-year-old woman with a diffuse midline glioma, H3 K27M-mutant, WHO grade IV with a remarkable longitudinal extension, extending from the cervical myelon to the basal ganglia. On imaging, the lesion was predominantly suggestive of inflammatory oedema, and it was clinically associated with progressive hemi- and later tetraparesis with severe autonomic and bulbar symptoms. Laboratory examinations suggested a generalized inflammatory process; however, neither infectious nor autoimmune aetiology could be confirmed. Biopsy was deemed unfeasible given the critical localization. Presuming a seronegative autoimmune encephalomyelitis, high-dose corticosteroid therapy and plasma exchanges were conducted, resulting in a modest but transient relief. The patient passed away two months after hospitalization. Neuropathological examination of the lesion revealed a high-grade diffuse glioma with $\mathrm{H} 3$ K27M mutation (grade IV). Although originally considered as a paediatric entity, our case confirms reports from recent years that diffuse midline gliomas, H3 K27M-mutant, WHO grade IV can occur in adults, even among the elderly, and can mimic inflammatory alterations, posing diagnostic difficulty. Our case is one of the oldest patients reported with this pathology, the oldest with an extensive diffusely infiltrating growth pattern, and with the most extensive lesion reported in adulthood.
\end{abstract}

Key words: diffuse intrinsic pontine glioma, diffuse midline glioma, elderly, histone, K27M.

\section{Introduction}

Diffuse midline glioma, H3 K27M-mutant, World Health Organization (WHO) grade IV represents a distinct entity of diffuse gliomas with a predominantly paediatric presentation and remarkably poor prog- nosis [11,21]. Recurrent somatic K27M point mutation (i.e., lysine to methionine substitution at codon 27) in these tumours can affect either the H3F3A or the HIST1H3B genes, encoding histone $\mathrm{H} 3$ variants $\mathrm{H} 3.3$ and $\mathrm{H} 3.1$, respectively, in a mutually exclusive fashion,

Communicating author

Levente Szalardy, MD, PhD, Department of Neurology, Faculty of Medicine, Albert Szent-Györgyi Clinical Center, University of Szeged, Semmelweis u. 6, H-6725 Szeged, Hungary, e-mail: szalardy.levente@med.u-szeged.hu 
with the H3.3 K27M mutation being more common [21]. In addition, recurrent $\mathrm{H} 3.3$ mutations at codon 34 with glycine to arginine or valine substitutions (G34R/V) have also been identified in paediatric as well as adult diffuse gliomas; however, H3.3 G34R/Vmutant tumours are most predominantly hemispheric in location, are less frequent, and are associated with somewhat better prognosis, compared to patients with a tumour harbouring a K27M mutation [20,21].

Although diffuse midline glioma, H3 K27M-mutant, WHO grade IV has been historically regarded as a strictly paediatric condition [21], case reports, case series, and larger studies have recently been published with adult and rarely with elderly cases (Table I) $[12,15]$. Here we report one of the oldest patients having so far been reported with an immunohistochemically confirmed diffuse midline glioma, H3 K27M-mutant (WHO grade IV), with an exceptionally extensive longitudinal spread (extending from the cervical myelon to the basal ganglia) and non-specific imaging characteristics clinically mimicking an inflammatory process. The report was prepared in accordance with the CARE (CAse REport) guidelines [10]. All procedures performed were in accordance with the ethical standards of the 1964 Helsinki declaration and its later amendments. The publication of the case report was approved by the Regional Human Biomedical Research Ethics Committee of the University of Szeged under registration number 44/2016. The next-of-kin provided written informed consent for the publication of the case.

\section{Case presentation \\ Clinical summary}

A 73-year-old woman of Caucasian ethnicity was admitted to our neurology department from a rural hospital with 1-month history of progressive neurological signs and symptoms. Her complaints started with tingling of the right side of her mouth and tongue, and urinary incontinence. Two weeks later, she developed weakness of the facial muscles and extremities on the left side, followed by swallowing disturbance and faecal incontinence shortly before referral to our ward. Her past medical history was relevant for hypertension, a thyroid nodule, and chronic cholecystitis. The family history included breast cancer in her mother.

On physical examination on admission, the patient presented with left-sided central facial pal- sy, severe dysarthria, severe dysphagia, absent gag reflex, moderate left-sided hemiparesis with bilateral pyramidal signs both on the upper and lower limbs (positive Hoffmann's sign, Trömner's sign, inverted radial reflex, and Babinski reflex), a slight cerebellar ataxia in the right limbs, and a right-sided facial paraesthesia. The deep tendon reflexes were brisk in the upper but lost in the lower extremities. The patient was unable to sit and walk.

The multiple cranial nerve symptoms with the bilateral corticospinal tract involvement were suggestive of a central nervous system (CNS) lesion involving the pontomedullary brainstem. A cranial computed tomography (CT) had already been performed in the other institution with no abnormality detected. The magnetic resonance imaging (MRI) of the head and the neck performed in our hospital revealed a longitudinally extending confluent $\mathrm{T} 2$ - and fluid-attenuated inversion recovery (FLAIR)-hyperintense lesion, starting from the second segment of the cervical spinal cord, extending through the medulla, the pons, the cerebral peduncles, and the internal capsules bilaterally, and extending to the right pallidum (Fig. 1). The lesion demonstrated no apparent Gadolinium contrast enhancement. On MR angiography, the large arteries were intact, and the diffusion-weighted image (DWI) was not indicative of ischaemic alteration (not shown). The presentation of the lesion was suggestive of interstitial oedema, raising the suspicion of an inflammatory alteration (i.e., brainstem encephalitis (including Bickerstaff brainstem encephalitis (BBE)), neuromyelitis optica spectrum disorder (NMOSD), or CNS vasculitis (including Behçet's disease)) or an infiltrative tumour.

Laboratory examinations from the blood demonstrated elevated inflammatory parameters suggestive of systemic inflammation (i.e., elevated C-reactive protein (CRP) level and sedimentation rate, slightly elevated levels of complements 3 and 4, and oligoclonal banding of gamma globulins, accompanied by normal white blood cell count) and uniformly elevated liver enzymes. Small-vessel vasculitis with involvement of the cerebral vessels was addressed, but the serum was negative for typical autoantibodies (including anti-neutrophil cytoplasmic antibodies (ANCA), anti-myeloperoxidase (MPO) and anti-proteinase 3 (PR3) antibodies, as well as autoantibodies of connective-tissue diseases). The elevated activated partial thromboplastin time (aPTT) and the results of the mixing test suggested the presence of 


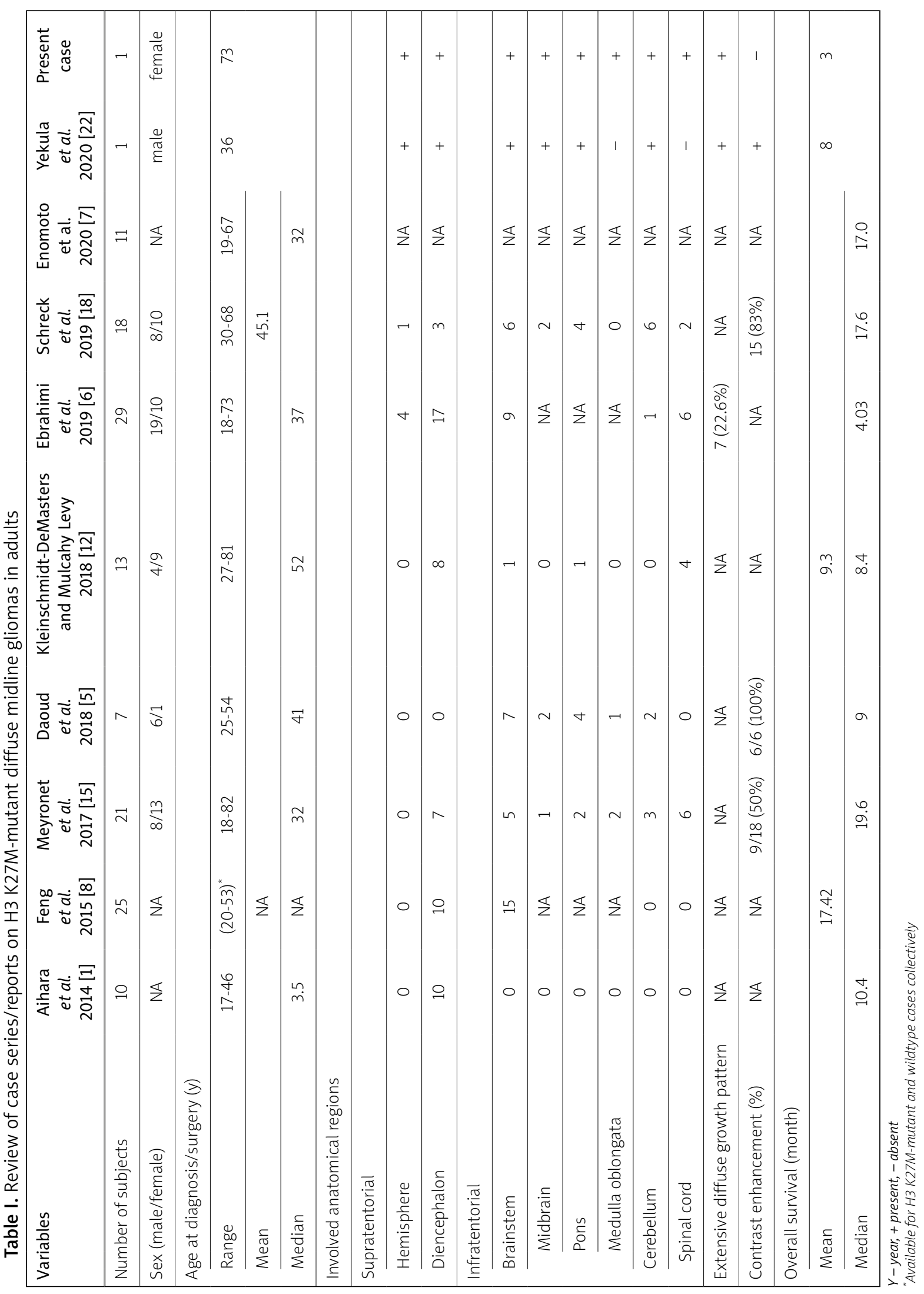



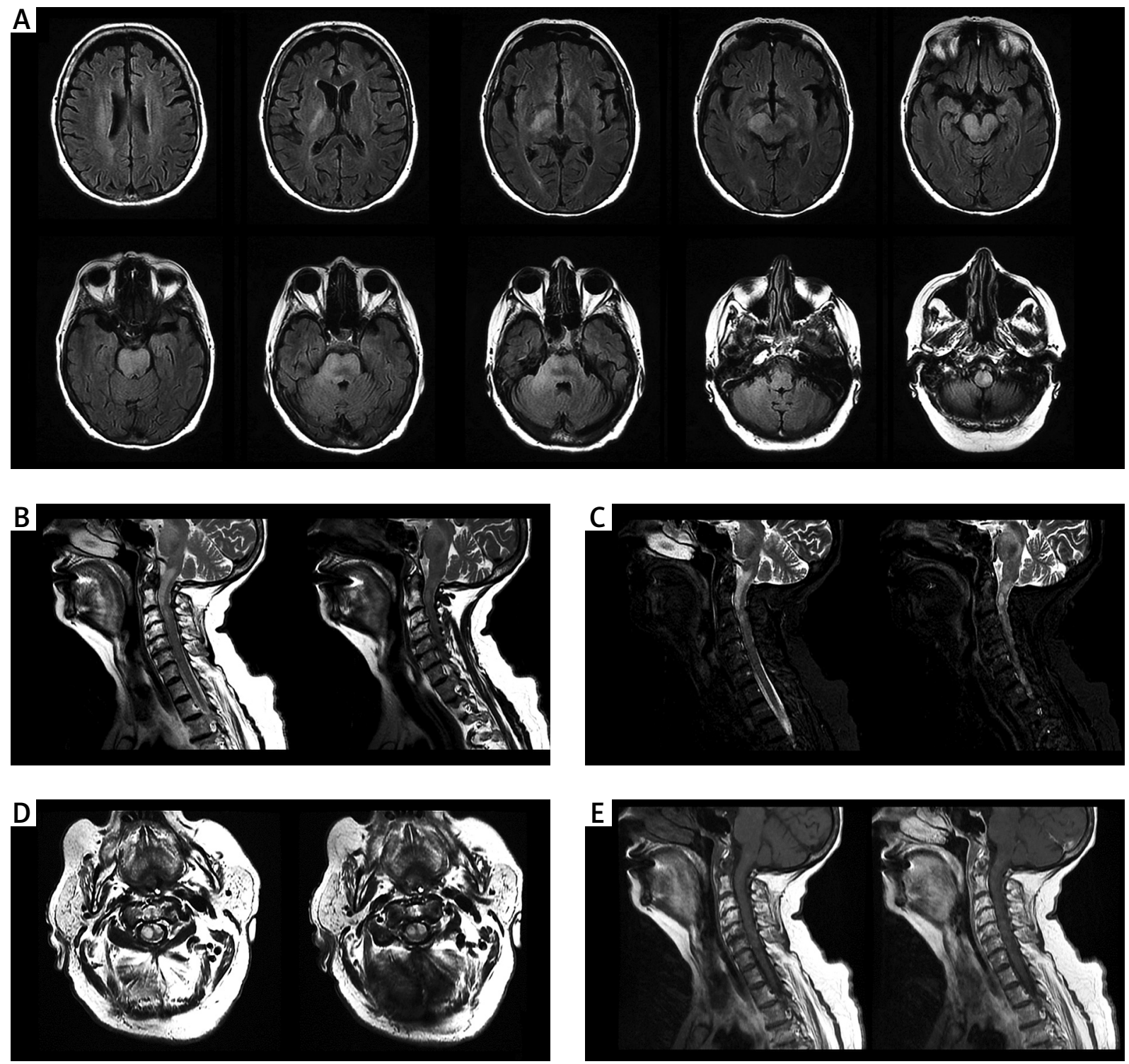

Fig. 1. Representative magnetic resonance imaging (MRI) scans of the elderly female patient with longitudinally extending diffuse midline glioma mimicking encephalomyelitis. A) Axial cranial MRI fluid-attenuated inversion recovery (FLAIR) images with hyperintense lesion affecting the right-sided globus pallidus (with a suspicious vague hyperintensity in the overlying white matter as well), the internal capsules, the cerebral peduncles, the pontine basis and the cerebellar peduncles with right-sided predominance, and the medulla oblongata with a left-sided predominance. B) Sagittal cervical MRI T2 fast recovery fast spin-echo (FRFSE) images and C) corresponding short tau inversion recovery (STIR) images of the cervical spine demonstrating a longitudinally extensive hyperintense lesion suggestive of interstitial oedema. D) The lesion demonstrates a longitudinally extending spread toward the upper cervical segments in forms of two distinct columns predominantly in the aspect of the anterior and lateral funiculi in axial T2 FRFSE images. E) No apparent Gadolinium contrast enhancement can be seen within the lesion (sagittal cervical T1 fast spin-echo (FSE) MRI image without contrast in the left and with contrast in the right). 
lupus anticoagulant; however, this alteration could as well have arisen secondary to the prophylactically administered low-molecular weight heparin (LMWH) therapy, limiting its interpretation [16]. No other anti-phospholipid antibodies were positive (including screening for anti-cardiolipin and anti- $\beta$-2glycoprotein 1 antibodies). The routine cerebrospinal fluid (CSF) investigation did not disclose any alteration (with a normal cell count and protein level), no signs of intrathecal immunoglobulin G (IgG) or immunoglobulin $M(\operatorname{IgM})$ synthesis was detected by nephelometry, and oligoclonal bands were not present in the CSF (contrasting with the serum). No infectious agents were detected in the CSF (neither bacteria or fungus in the cultures nor neurotropic viruses (including herpes simplex virus (HSV) 1 and 2, Epstein-Barr virus (EBV), cytomegalovirus (CMV), enterovirus, and parechovirus) or Toxoplasma gondii by real-time polymerase chain reaction (RT-PCR)), and no signs of active infection with neurotropic viruses (including HSV 1 and 2, varicella zoster virus, EBV, CMV, hepatitis $B$ and $C$ virus, human immunodeficiency virus 1 and 2, West-Nile virus, and tick-borne encephalitis virus) or Treponema pallidum could be detected by blood serology. Likewise, autoimmune encephalitis-associated antibodies, onconeural auto-antibodies, anti-glutamic acid decarboxylase (GAD) antibody, anti-aquaporin-4 (AQP4) antibody, and anti-myelin oligodendrocyte glycoprotein (MOG) antibody were not detected in the CSF (neither from the serum in the case of the last two). Based on the presence of corticospinal signs and limb ataxia, the Guillain-Barré syndrome (GBS)-variant BBE also turned up as a possible diagnosis (even though ophthalmoplegia was not seen); therefore, anti-ganglioside GQ1b protein was tested, which was again negative. The patient was referred to the neurosurgery to facilitate histological diagnosis, but biopsy was not taken as the procedure was deemed overly hazardous due to the critical localization of the lesion. With the presumptive diagnosis of an inflammatory process in the CNS (with some of the above laboratory test results still pending), the administration of high-dose intravenous methyl-prednisolone (a total of $2.5 \mathrm{~g}$ ) with empirical antibiotics (for the concomitant infections) was conducted, which resulted in a transient improvement in the patient's state.

On follow-up cranial MRI scans (2 and 7 weeks after the initial one), no change in the extent of the lesion could be detected intracranially, with only the 7-week follow-up cervical MRI demonstrating a slight progression to the caudal direction (not shown). Neuroradiological consultations consistently considered the lesion as inflammatory. The MRI spectroscopy suggested increased metabolism within the lesion, with no further specification.

Over time, the hemiparesis became more severe and later spread to the other side as well, and an almost complete anarthria and eventually a decreased level of consciousness developed. Another cycle of pulse corticosteroid therapy was given $(2.5 \mathrm{~g})$, without any therapeutic success at this trial. As an ultimum refugium, the patient ex juvantibus received plasma exchange four times, but it also had no positive result. Eventually, after 2 months of hospitalization and some 3 months of symptom onset, the patient passed away as a consequence of recurrent nosocomial infections superimposed on the severe bed-ridden state.

\section{Neuropathological assessment}

\section{Material and methods}

The brain was fixed in buffered formalin for 4 weeks. After fixation, coronal sections were performed at $0.7-\mathrm{mm}$ interval and several samples were taken from macroscopically identified alterations as well as from unremarkable distant areas. Haematoxylin-eosin-stained slides and immunostainings were performed according to standard protocols on 4- $\mu \mathrm{m}$ formalin-fixed paraffin-embedded sections. The following antibodies were used: anti-glial fibrillary acidic protein (GFAP, mouse monoclonal, clone ASTRO6, 1:5000, Thermo Fisher Scientific, Waltham, MA, USA), anti-histone H3 (mutated K27M, rabbit monoclonal, clone EPR18340, $1: 100$, Abcam, Cambridge, UK), anti-isocitrate dehydrogenase 1 (IDH1) (R132H mutant, mouse monoclonal, clone IHC132, 1 : 200, GenomMe, Richmond, BC, Canada), antiKi-67 (rabbit monoclonal, clone SP6, $1: 100$, Hisztopatológia Ltd., Pécs, Hungary), and anti-p53 (mouse monoclonal, clone DO7, 1 : 300, Sigma-Aldrich, St. Louis, MO, USA). The DAKO EnVision detection kit, peroxidase/3,3'-diaminobenzidine (DAB), rabbit/ mouse (Dako, Glostrup, Denmark) was used for visualization of antibody reactions. Photographs were taken using the CaseViewer software of a Pannoramic MIDI digital slide scanner (3D Histech, Budapest, Hungary). 

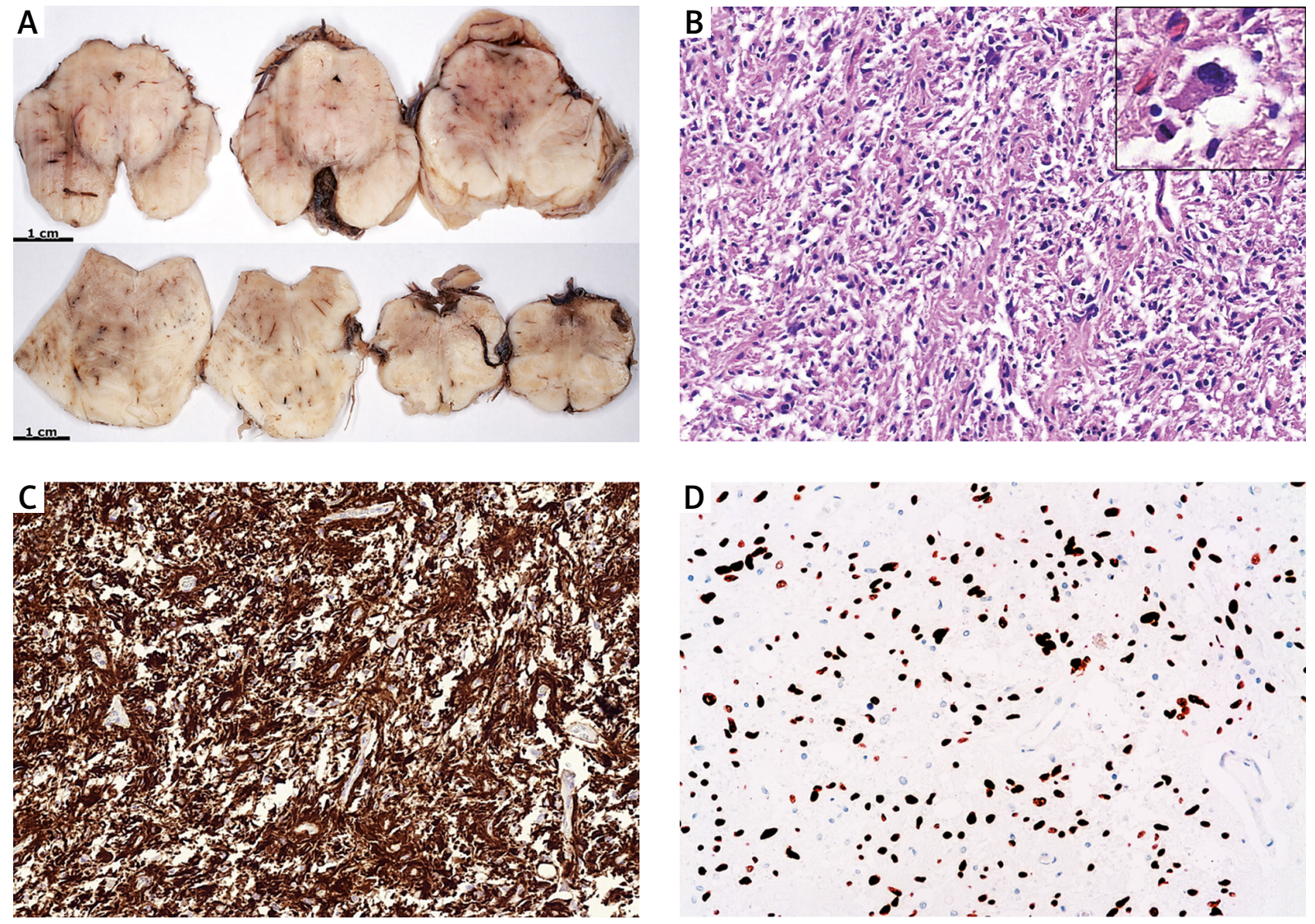

Fig. 2. A) Gross neuropathological examination of coronal brain slices reveals a diffusely infiltrating tumour predominantly involving the right cerebral peduncle, the basis of the pons, and the medulla oblongata. B) Haematoxylin-eosin staining of the cerebral peduncle demonstrates a hypercellular glial tumour with a predominant fibrillary background, composed of highly pleomorphic cells with indistinct cell borders, variable amount of eosinophilic cytoplasm, and oval to irregular hyperchromatic nuclei. Mitotic figures are frequent and occasional bizarre multinucleated cells are also present (inset). C) The tumour shows diffuse immunopositivity for glial fibrillary acidic protein (GFAP), and D) a strong nuclear labelling for K27M-mutant histone $\mathrm{H} 3$, consistent with the diagnosis of diffuse midline glioma, H3 K27M-mutant, WHO grade IV.

\section{Neuropathological findings}

On gross neuropathological examination, the cerebral hemispheres were symmetrical with no evidence of focal lesion on the convex lateral surface. At the base of the brain, the pons and the medulla oblongata were mildly enlarged with slight asymmetry. There was no evidence of uncal or tonsillar herniation. The basal arteries and cranial nerves were unremarkable. Coronal sections of the brainstem showed white, slightly blurred areas which were firm to touch, suggesting a diffusely infiltrating tumour, predominantly involving the cerebral peduncles (less apparently on the left side), the basis of the pons, and the right internal capsule (Fig. $2 \mathrm{~A}$ ). The infiltrat- ed areas were slightly enlarged with mild distortion of the anatomical structures.

Histological work-up revealed a focally hypercellular glial tumour diffusely infiltrating the cerebral peduncles, the basis of the pons, the right cerebellar peduncle, and the medulla oblongata. Infiltrating tumour cells were also suspected along the white matter tract of the internal capsules. The tumour consisted of pleomorphic astrocytic cells with significant mitotic activity and some bizarre multinucleated giant cells (Fig. 2B). There was only very mild microvascular proliferation noted with no characteristic glomeruloid blood vessels. The infiltrated neurons were remarkably intact and no necrosis was identified in the tumour. Immunohistochemistry showed 
strong diffuse positivity for GFAP in the tumour (Fig. 2C). The IDH1 (R132H) mutant clone was negative, most in keeping with an IDH-wildtype glioma at the age of the patient. No p53 overexpression was seen. The estimated proliferation index by Ki67 was about $8-9 \%$. In addition, most of the tumour cells showed strong nuclear labelling for K27M-mutant histone H3 (H3 K27M), which confirmed the diagnosis of a diffuse midline glioma, H3 K27M-mutant, WHO grade IV (Fig. 2D).

\section{Discussion}

This case report demonstrates the diagnostic difficulty and the unexpected neuropathological diagnosis of a rapidly progressing pathological lesion, which diffusely affected critical anatomical regions in the midline of the CNS, including the brainstem, in an elderly woman. The initial MRI images showed that the pathology was associated with interstitial oedema with an irregularly spreading confluent character, opening the question whether the oedema was due to a primary inflammation or a neoplasm (including a high-grade glioma in a diffusely infiltrating pattern (i.e., without a discrete tumour mass, reminiscent in this respect of the gliomatosis cerebri growth pattern, albeit not meeting its criteria due to the lack of multilobar involvement) [9] or a primary CNS lymphoma in a lymphomatosis cerebri pattern [13] in the differential diagnosis). Due to the critical localization of the lesion, biopsy was not attempted by the neurosurgery. After initiating a comprehensive evaluation for a range of inflammatory conditions including autoimmune small-vessel CNS vasculitides, CNS infections, paraneoplastic/ autoimmune encephalitides, NMOSD, and BBE, highdose corticosteroid therapy was administered with empirical and, subsequently, targeted antibiotics for systemic infections. The first cycle of pulse corticosteroid therapy moderately alleviated some of the symptoms, which supported the presumption of an immunological process; however, we did not manage to identify disease-specific autoantibodies either from the serum or from the CSF. This initial therapeutic response to the first trial of pulse corticosteroid therapy can be presumed to be due to a decrease in tumour-associated oedema, retrospectively. On the basis of the serial negative results and the normal cell count and protein concentration in the CSF, a potential seronegative CNS condition of immunological origin became a less likely albeit the sole potentially treatable diagnosis (in fact the patient met the criteria of seronegative NMOSD). Though no investigation was performed that could have helped to differentiate between inflammatory and neoplastic origin (with the MR spectroscopy revealing aspecific hypermetabolism indicative of both), the latter seemed more likely in an exclusionary perspective, especially after the lack of symptomatic relief after the second corticosteroid trial and the repeated plasma exchanges. An 18F-fluorodeoxyglucose (FDG) positron emission tomography (PET) CT and 11C-methionine (MET) PET-CT were also considered to address a neoplastic origin; however, the broad literature on the limitations of these methods in differentiating inflammatory alterations from malignancies [4,17], the already very advanced clinical state of the patient at this stage, together with the inability to obtain in vivo histological diagnosis and thus to administer targeted therapy in case of a potential supportive finding overall resulted in a decision to omit this examination.

The definite diagnosis was built up by post mortem histopathology and immunohistochemistry, revealing a high-grade glioma. Given the midline localization of the tumour, examination for the histone gene mutation $\mathrm{H} 3 \mathrm{~K} 27 \mathrm{M}$ was conducted immunohistochemically, which yielded positive results, allowing the establishment of the diagnosis of diffuse midline glioma, H3 K27M-mutant, WHO grade IV. This method is sensitive for both H3.3 and H3.1 K27M mutations and has been documented to demonstrate $100 \%$ correspondence with the findings of DNA sequencing $[2,6]$. Based on the fact that only post mortem, formalin-fixed, paraffin-embedded tissue was available (yielding a high possibility of diagnostic failure for molecular analysis) and that the definite diagnosis of this glioma entity can be established on immunohistochemical grounds alone (reflected also by its exclusive use in a number of recent larger-scale studies $[5,12,18,19])$, further specification of the locus of the $\mathrm{H} 3 \mathrm{~K} 27 \mathrm{M}$ mutation by molecular techniques was not performed.

This type of diffuse midline gliomas was classified as a new entity in the 2016 WHO classification of brain tumours as diffuse midline glioma, H3 K27M-mutant, WHO grade IV [14]. Diffuse midline gliomas had originally been described in children and were historically referred to as paediatric diffuse intrinsic pontine gliomas (DIPGs). Molecular analysis revealed that some $70-80 \%$ of DIPGs carry H3 K27M 
mutation, and are typically IDH-wildtype [11,21]. Tumours harbouring this mutation have remarkably poor prognosis despite treatment with adequate chemotherapy (temozolomide) and radiotherapy [11,15]; though this association in adult cases has recently been questioned $[7,18]$. The poorer prognosis compared to $\mathrm{H} 3$ wildtype gliomas may be related to the specific point mutation resulting in gliomagenesis through epigenetic changes. Although originally described as a paediatric entity, more recently, diffuse midline gliomas, H3 K27M-mutant, WHO grade IV have been reported in young and even in elderly adults, with case reports and series appearing in the literature only in the past few years [1,5-8, $2,15,18,22]$ (Table I). To our knowledge, only two cases have so far been reported that were older than our patient, an 82-year-old female patient with an isolated thalamic presentation and a 5.5-month survival [15], and an 81-year-old female patient with an isolated hypothalamic presentation and a 1.9-month survival [12]. As for the same 73 years of age, 2 male patients have so far been reported, with an isolated spinal cord involvement, however [6].

A longitudinally extensive spread of paediatric diffuse midline gliomas has been well documented, including tumours harbouring an $\mathrm{H} 3 \mathrm{~K} 27 \mathrm{M}$ mutation, with some of the reported autopsy cases presenting with a lesion extending from the medulla to the frontal lobe [3]. Although a longitudinally extensive diffusely infiltrating growth pattern of such tumours in adults has also recently been documented $[6,22]$, such an extensive diffuse midline glioma, H3 K27M-mutant, WHO grade IV (extending from the cervical myelon to the basal ganglia) in adulthood, to our knowledge, is unique.

\section{Conclusions}

Though originally described as a paediatric entity, diffuse midline glioma, H3 K27M-mutant, WHO grade IV can occur in adults, even in the elderly. The longitudinally extensive topography and occasionally non-specific imaging characteristics (as in our case) can mimic inflammatory CNS processes and represent a diagnostic challenge, especially when biopsy is deemed unfeasible due to critical localization of the lesion.

\section{Acknowledgements}

LS was supported by the János Bolyai Research Scholarship of the Hungarian Academy of Sciences, the ÚNKP-18-4 New National Excellence Program of the Ministry of Human Capacities, and the ÚNKP-19-4 New National Excellence Program of the Ministry for Innovation and Technology.

\section{Disclosure}

The authors report no conflict of interest.

\section{References}

1. Aihara K, Mukasa A, Gotoh K, Saito K, Nagae G, Tsuji S, Tatsuno K, Yamamoto S, Takayanagi S, Narita Y, Shibui S, Aburatani H, Saito N. H3F3A K27M mutations in thalamic gliomas from young adult patients. Neuro Oncol 2014; 16: 140-146.

2. Bechet D, Gielen GG, Korshunov A, Pfister SM, Rousso C, Faury D, Fiset PO, Benlimane N, Lewis PW, Lu C, David Allis C, Kieran MW, Ligon KL, Pietsch T, Ellezam B, Albrecht S, Jabado N. Specific detection of methionine 27 mutation in histone 3 variants (H3K27M) in fixed tissue from high-grade astrocytomas. Acta Neuropathol 2014; 128: 733-741.

3. Buczkowicz P, Bartels U, Bouffet E, Becher O, Hawkins C. Histopathological spectrum of paediatric diffuse intrinsic pontine glioma: diagnostic and therapeutic implications. Acta Neuropathol 2014; 128: 573-581.

4. Cheong SJ, Lee CM, Kim EM, Lim ST, Sohn MH, Jeong HJ. The effect of PPAR-gamma agonist on (18)F-FDG PET imaging for differentiating tumors and inflammation lesions. Nucl Med Biol 2015; 42: 85-91.

5. Daoud EV, Rajaram V, Cai C, Oberle RJ, Martin GR, Raisanen JM, White CL, 3rd, Foong C, Mickey BE, Pan E, Hatanpaa KJ. Adult brainstem gliomas with $\mathrm{H} 3 \mathrm{~K} 27 \mathrm{M}$ mutation: radiology, pathology, and prognosis. J Neuropathol Exp Neurol 2018; 77: 302-311.

6. Ebrahimi A, Skardelly M, Schuhmann MU, Ebinger M, Reuss D, Neumann M, Tabatabai G, Kohlhof-Meinecke P, Schittenhelm J. High frequency of H3 K27M mutations in adult midline gliomas. J Cancer Res Clin Oncol 2019; 145: 839-850.

7. Enomoto T, Aoki M, Hamasaki M, Abe H, Nonaka M, Inoue T, Nabeshima K. Midline glioma in adults: clinicopathological, genetic, and epigenetic analysis. Neurol Med Chir (Tokyo) 2020; 60: 136-146.

8. Feng J, Hao S, Pan C, Wang Y, Wu Z, Zhang J, Yan H, Zhang L, Wan $\mathrm{H}$. The H3.3 K27M mutation results in a poorer prognosis in brainstem gliomas than thalamic gliomas in adults. Hum Pathol 2015; 46: 1626-1632.

9. Fernandez-Vega I, Quirk J, Norwood FL, Sibtain NA, Laxton R, Bodi I. Gliomatosis cerebri type 1 with extensive involvement of the spinal cord and BRAF V600E mutation. Pathol Oncol Res 2014; 20: 215-220.

10. Gagnier JJ, Kienle G, Altman DG, Moher D, Sox H, Riley D. The CARE Guidelines: consensus-based clinical case reporting guideline development. Glob Adv Health Med 2013; 2: 38-43.

11. Khuong-Quang DA, Buczkowicz P, Rakopoulos P, Liu XY, Fontebasso AM, Bouffet E, Bartels U, Albrecht S, Schwartzentruber J, Letourneau L, Bourgey M, Bourque G, Montpetit A, Bourret G, Lepage P, Fleming A, Lichter P, Kool M, von Deimling A, Sturm D, Korshunov A, Faury D, Jones DT, Majewski J, Pfister SM, Jaba- 
do N, Hawkins C. K27M mutation in histone H3.3 defines clinically and biologically distinct subgroups of pediatric diffuse intrinsic pontine gliomas. Acta Neuropathol 2012; 124: 439-447.

12. Kleinschmidt-DeMasters BK, Mulcahy Levy JM. H3 K27M-mutant gliomas in adults vs. children share similar histological features and adverse prognosis. Clin Neuropathol 2018; 37: 53-63.

13. Li L, Rong JH, Feng J. Neuroradiological features of lymphomatosis cerebri: A systematic review of the English literature with a new case report. Oncol Lett 2018; 16: 1463-1474.

14. Louis DN, Perry A, Reifenberger G, von Deimling A, FigarellaBranger D, Cavenee WK, Ohgaki H, Wiestler OD, Kleihues P, Ellison DW. The 2016 World Health Organization Classification of Tumors of the Central Nervous System: a summary. Acta Neuropathol 2016; 131: 803-820.

15. Meyronet D, Esteban-Mader M, Bonnet C, Joly MO, Uro-Coste E, Amiel-Benouaich A, Forest F, Rousselot-Denis C, Burel-Vandenbos F, Bourg V, Guyotat J, Fenouil T, Jouvet A, Honnorat J, Ducray F. Characteristics of H3 K27M-mutant gliomas in adults. Neuro Oncol 2017; 19: 1127-1134.

16. Pengo V, Tripodi A, Reber G, Rand JH, Ortel TL, Galli M, De Groot PG. Update of the guidelines for lupus anticoagulant detection. Subcommittee on Lupus Anticoagulant/Antiphos pholipid Antibody of the Scientific and Standardisation Committee of the International Society on Thrombosis and Haemostasis. J Thromb Haemost 2009; 7: 1737-1740.

17. Rosenbaum SJ, Lind T, Antoch G, Bockisch A. False-positive FDG PET uptake - the role of PET/CT. Eur Radiol 2006; 16: 1054-1065.

18. Schreck KC, Ranjan S, Skorupan N, Bettegowda C, Eberhart CG, Ames HM, Holdhoff M. Incidence and clinicopathologic features of H3 K27M mutations in adults with radiographicallydetermined midline gliomas. J Neurooncol 2019; 143: 87-93.

19. Solomon DA, Wood MD, Tihan T, Bollen AW, Gupta N, Phillips JJ, Perry A. Diffuse midline gliomas with histone H3-K27M mutation: a series of 47 cases assessing the spectrum of morphologic variation and associated genetic alterations. Brain Pathol 2016; 26: 569-580.

20. Sturm D, Witt H, Hovestadt V, Khuong-Quang DA, Jones DT, Konermann C, Pfaff E, Tonjes M, Sill M, Bender S, Kool M, Zapatka M, Becker N, Zucknick M, Hielscher T, Liu XY, Fontebasso AM, Ryzhova M, Albrecht S, Jacob K, Wolter M, Ebinger M, Schuhmann MU, van Meter T, Fruhwald MC, Hauch H, Pekrun A, Radlwimmer B, Niehues T, von Komorowski G, Durken M, Kulozik AE, Madden J, Donson A, Foreman NK, Drissi R, Fouladi M, Scheurlen W, von Deimling A, Monoranu C, Roggendorf W, Herold-Mende C, Unterberg A, Kramm CM, Felsberg J, Hartmann C, Wiestler B, Wick W, Milde T, Witt O, Lindroth AM, Schwartzentruber J, Faury D, Fleming A, Zakrzewska M, Liberski PP, Zakrzewski K, Hauser P, Garami M, Klekner A, Bognar L, Morrissy S, Cavalli F, Taylor MD, van Sluis P, Koster J, Versteeg R, Volckmann R, Mikkelsen T, Aldape K, Reifenberger G, Collins VP, Majewski J, Korshunov A, Lichter P, Plass C, Jabado N, Pfister SM. Hotspot mutations in H3F3A and IDH1 define distinct epigenetic and biological subgroups of glioblastoma. Cancer Cell 2012; 22: 425-437.

21. Wu G, Broniscer A, McEachron TA, Lu C, Paugh BS, Becksfort J, Qu C, Ding L, Huether R, Parker M, Zhang J, Gajjar A, Dyer MA,
Mullighan CG, Gilbertson RJ, Mardis ER, Wilson RK, Downing JR, Ellison DW, Baker SJ. Somatic histone H3 alterations in pediatric diffuse intrinsic pontine gliomas and non-brainstem glioblastomas. Nat Genet 2012; 44: 251-253.

22. Yekula A, Gupta M, Coley N, Sang UH. Adult H3K27M-mutant diffuse midline glioma with gliomatosis cerebri growth pattern: Case report and review of the literature. Int I Surg Case Rep 2020; 68: 124-128. 